\title{
Effect of different self-selected walking speeds in leveling of body center of mass, mechanical work and energy in healthy children
}

\author{
MARIANA CARRIQUIRY ${ }^{1}$, VALENTINA SILVA-PEREYRA ${ }^{2}$, \\ DANIEL JEREZ-MAYORGA ${ }^{3 *}$, GABRIEL FÁBRICA ${ }^{1}$ \\ ${ }^{1}$ Faculty of Medicine, Universidad de la República, Montevideo, Uruguay. \\ ${ }^{2}$ Instituto Superior de Educación Física, Universidad de la República, Paysandú, Uruguay. \\ ${ }^{3}$ Faculty of Rehabilitation Sciences, Universidad Andres Bello, Santiago, Chile.
}

\begin{abstract}
Purpose: The aim of the study was to analyze the general kinematics of the cycle, leveling of the center of mass and inverted pendulum model in school-age children when they walk at three different auto-selected speeds. Methods: The kinematics of walking cycle, angular actions that contribute to reducing the vertical displacement of body center of mass (pelvis, hip, knee and ankle) and pendulumlike determining variables (mechanical work, pendulum-like recovery and congruity percentage), were analyzed in children for three different self-selected speeds. Differences for each variable with the speed were tested by ANOVA with Bonferroni post-hoc analysis. Omega squared $\left(\omega^{2}\right)$ was calculated for the values of the effect sizes. Results: None of the angular variables associated with the leveling of the vertical trajectory of body center of mass changed. Likewise, recovery and congruity percentage presented values similar to those obtained in previous studies and did not show significant changes with the speeds. Conclusions: Nevertheless, changes in horizontal mechanical work and cycle phases, indicate that at some point during the cycle the mechanical energy transfer may have been affected for speed changes. Our results warn about the implication that small changes in the speed during functional evaluations of gait in children may have.
\end{abstract}

Key words: gait analysis, clinical measurements, biomechanical modeling

\section{Introduction}

The gait pattern depends on the coordination of a set of biomechanical features, an understanding of the role of these factors in the generation of a healthy gait, can provide insight into gait deficits study [17], [24], [35]. In particular, the mechanical energy changes (i.e., the work), required to maintain the overall motion have received considerable attention because they potentially affect the metabolic expenditure [26].

The sum of two items composes the total mechanical work, the external mechanical work $\left(W_{e x t}\right)$ needed to raise and accelerate the body center of mass $(\mathrm{CoM})$ within the environment [6], and the internal mechani- cal work $\left(W_{\text {int }}\right)$ needed to accelerate the limbs in respect to CoM [6], [14]. Two strategies associated with $W_{\text {ext }}$ contribute to optimize gait minimizing the energetic cost; level the vertical trajectory of the CoM and taking advantage of the inverted pendulum mechanism [4], [12]. The first is associated with a set of combined movements of lower limbs and the pelvis [9], [18], [27], [33]. The second states that in each step, legs behave like an inverted pendulum in the sagittal plane, which enables the CoM to conserve mechanical energy [4], [26].

The actions that contribute to leveling of the trajectory of the CoM during gait include rotation of the pelvis in the transverse plane, rotation of pelvis in frontal plane, abduction/adduction of hip in in frontal

\footnotetext{
* Corresponding author: Daniel Jerez-Mayorga, Faculty of Rehabilitation Sciences, Universidad Andres Bello, Santiago, Chile. Phone: +56977697643, e-mail: daniel.jerez@unab.cl

Received: April 14th, 2021

Accepted for publication: August 16th, 2021
} 
plane, knee flexion in medium stance, flexion of knee in swing and rolling action of ankle [33]. Those actions can be visualized through different specific variables. However, it is noteworthy that analysis has a correspondence with clinical evaluations in children. In this sense, some variables that are part of the gait deviation index represent a good alternative [16], [30]. Meanwhile, the adjustment to the pendulum mechanism is classically evaluated through percentage of energy recovery $(R)$ and percentage of congruence (\%Cong) [3], [33]. However, $W_{\text {ext }}$ explains only approximately $50 \%$ of the work done in walking [11], [25], [34], therefore, the evaluation of $W_{\text {int }}$ is essential for the functional evaluation of walking.

Changes in gait speed can affect the amplitude and shape of the curves of angular values of lower limbs [26], [33] as different work components, $R$ and \%Cong. This clearly occurs if the speed changes are large, however, in real life, human's gaits habitually vary within a more limited range than used in most studies [21], [22].

Knowing whether variations occur in all or some of these variables with changes in speed within a range used in activities of daily living is of particular interest in children. For children aged 5-12, the speed is an even more influential factor than age in the determination of gait patterns [31]. This approach in children with typical development can provide insight for the assessment of children with pathological gait. It is possible that the changes in speed that children normally make do not imply a significant change in the variables associated with the leveling of the CoM or the pendular mechanism. However, if in any case there is a significant change, it is important to separate the effect of velocity from the one produced by a pathology.

To address these research gaps, the aim of the study was to analyze the general kinematics of the cycle, leveling of the center of mass and inverted pendulum model in school-age children when they walk at three different auto-selected speeds. We hypothesized that variations in speed within the range that children can self-select in life diary activities would induce significative changes in general kinematics of walking cycle and some angular values of lower limbs. However, based on all previous studies, these changes are not enough or they compensate each other so that pendulum mechanism do not vary.

\section{Materials and methods}

An observational, analytical, and transversal study based on a 3D reconstruction of the walk was carried out, where the sample was obtained by convenience. The general kinematics of walking cycle, pendulum-like determining variables $R$ and \%Cong, and angular values associated with the determinants that contribute to reducing the vertical displacement of CoM that are part of Gait Deviation Index (GDI) were analyzed. The study was carried out in three different self-selected speeds to provide elements for a more complete functional evaluation of locomotion in children.

\subsection{Subjects}

Ten healthy children ( 5 boys and 5 girls; age $=8.4$ \pm 1.0 years, height $=1.36 \pm 0.07 \mathrm{~m}$, body mass $=33.8$ $\pm 6.6 \mathrm{~kg}$ ), participated in this study after obtaining informed written consent from their parents or tutors. All procedures of this investigation were performed respecting the ethical norms concordant with the Declaration of Helsinki. The study was approved by the "Hospital de Clínicas Dr. Manuel Quintela" ethic committee (reference number 2014/21). The exclusion criteria were: musculoskeletal, cardiorespiratory, metabolic or neurological disorders that could affect gait or compromise the understanding of the proposal.

\subsection{Procedures}

The environment was previously conditioned at $21{ }^{\circ} \mathrm{C}$. Children were prepared and reflective markers were placed on 45 anatomical references to make a $3 \mathrm{D}$ reconstruction of the whole body: four markers on the head - left front head, right front head, left back head, right back head; five markers on the trunk -7 th Cervical Vertebrae, 10th Thoracic vertebrae, clavicle, xiphoid process of the sternum, half of the right scapula; eight markers on each upper limb - acromio-clavicular joint, shoulder rotation center, arm between the markers of the shoulder and the elbow, lateral epicondyle, forearm between the elbow and wrist markers, wrist lateral marker, wrist medial marker, under the head of the second metacarpal; four markers on the pelvis - left anterior superior iliac spine, right anterosuperior iliac spine, left posterosuperior iliac spine, right posterosuperior spine iliac; eight markers on each lower limb - greater trochanter of the femur, lateral distal third of the thigh, surface lateral epicondyle of the knee, over the distal third of the calf, lateral malleolus on an imaginary line passing through the transmaleolar axis, calcaneus, head of the second metatarsal, head of the fifth metatarsal.

Eight cameras (Bonita B10) operating at $100 \mathrm{~Hz}$ and connected to the Vicon Motion System (Oxford 
Metrics, Ltd., Oxford, UK) enabled the researchers to register the position kinematic data for each marker using Vicon's Nexus 2.4 software. Children walked on a surface of $10 \mathrm{~m}$ in length and $4 \mathrm{~m}$ in width without slope in 3 different self-selected speeds: intermediate speed (V1), slow speed (V2) and fast speed (V3). The three speeds were not predetermined or controlled during the captures, children regulated them in response to the following instructions: walk as you usually do, as if you are in a hurry or as if you want to slow down. This strategy was chosen in order to compare records within the range of daily chosen speeds. Five records were made for each subject in each speed and three cycles of each one were analyzed, obtaining fifteen data of each variable per subject for each speed.

The kinematic data were first low-pass filtered with a fourth-order zero-lag Butterworth filter with a cutoff frequency of $6 \mathrm{~Hz}$. Each stride data was processed using algorithm routines written in Matlab R2018 ${ }^{\circledR}$ (Mathworks, Inc.). To determine the different contacts and takeoffs, the vertical and antero-posterior velocity records of the markers of the feet were used. This method has been tested in our laboratory in conjunction with force platform records and allows for an accurate identification of events without conditioning the locomotion of the subject. The stride phases: initial double support, single support, final double support and swing were determined. Two types of variables, kinematic and associated with the variation of mechanical energy during movement, were calculated. The kinematic were divided in general kinematics of walking cycle and angular values. General variables were: mean speed (VM), cadence, stride length (LC), and relative duration of support and swing phases. Angular variables considered were selected considering two criteria, combined movements of the lower limb and pelvis segments that level the trajectory of the CoM [9], [27], [33] and values considered for the definition of GDI for clinical evaluations of children [30]. The angular variables selected were: pelvic rotation range $(\mathrm{Pr})$, maximum pelvic obliquity (Po), maximum abduction adduction of hip $(\mathrm{H})$, maximum knee flexion during stance $(\mathrm{K} 1)$, maximum knee flexion during swing (K2), ankle dorsiflexion in the initial contact (Aci), maximum dorsiflexion during stance (Am).

Regarding the variables associated with the variation of mechanical energy, we considered the variables associated with CoM energy reconversion $\left(W_{v}, W_{h}\right.$, $W_{\text {ext }}, R$ and \%Cong), $W_{\text {int }}$ and $W_{\text {tot }}$. Were $W_{h}$ is the horizontal external mechanical work, $W_{v}$ is the vertical work, and $W_{\text {ext }}$ is the external mechanical work. These work components correspond to homologous energy variations. During healthy level walking, the exchange between kinetic energy in the forward direction $\left(E_{h}\right)$ and potential energy more kinetic energy in the vertical direction $\left(E_{v}\right)$ results in a total mechanical energy $\left(E_{t}=E_{h}+E_{v}\right)$ of CoM, with a smaller change over the stride compared to the two components when taken separately [4], [26].

Segmental mass, segmental center of mass position and segmental radius of gyration were estimated from anthropometric tables proposed by Dempster [34]. Owing to the importance of accurately locating the CoM from the kinematic data, we developed a spatial model considering criteria of a previous work [23].
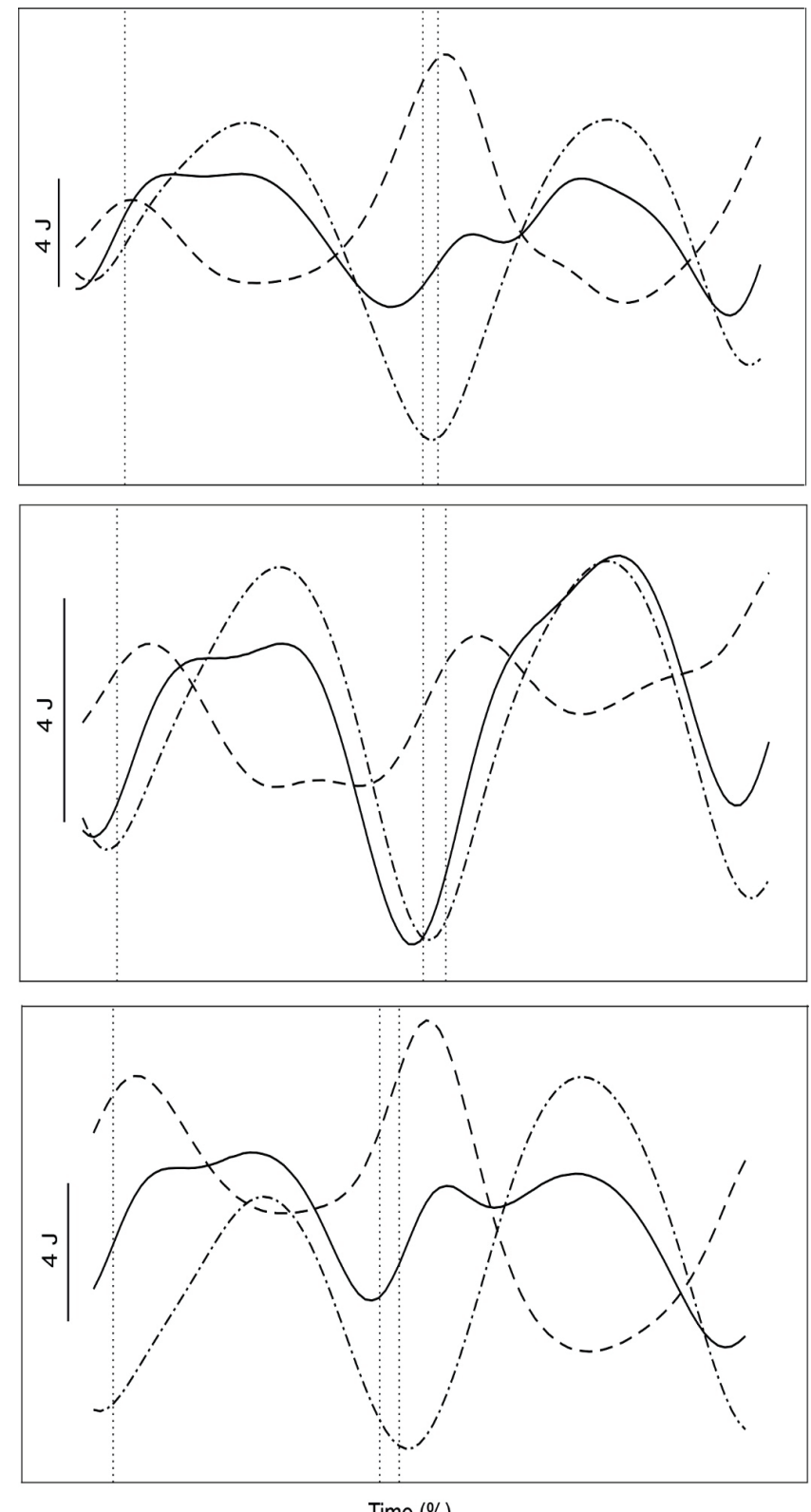

Fig. 1. Variations of $E_{h}$ (dashed line), $E_{v}$ (dashed point line) and $E_{t}$ (solid line) during the percentage of the cycle for V1 (above), V2 (in the middle) and V3 (below). Based on these variations, $W_{h}, W_{v}$ and $W_{\text {ext }}$ were determined to obtain $R$ 
$R$ was quantified as described by Cavagna and colleagues [4]:

$$
R=\left(\left(W_{v}+W_{h}-W_{\text {ext }}\right) /\left(W_{v}+W_{h}\right)\right) \times 100 .
$$

The \%Cong was calculated as the number of frames in which $d\left(E_{h}\right) / d t$ and $d\left(E_{v}\right) / d t$ have opposite sign divided by the total number of frames in a gait cycle $\times 100 \%$ [5].

$W_{\text {int }}$ was estimated as the sum of the increments of kinetic energy of each limb and of the head and trunk respect to CoM in the three planes of movement thorough the locomotion cycle. Energy transfer between the segments of the same limb was assumed. Once the data corresponding to the $W_{\text {int }}$ were obtained, the calculation of the $W_{t o t}$ was proposed as the sum of $W_{e x t}$ and $W_{\text {int }}$. More details of how the calculations are addressed can be found in the classic work [32].

\subsection{Statistical analysis}

Descriptive data is presented as mean and standard deviation (SD). The normal distribution of the data (Shapiro-Wilk test) and the homogeneity of variances (Levenes's test) were confirmed ( $p>0.05)$. For the main analysis, a repeated-measures analysis of variance (ANOVA) was conducted with Bonferroni posthoc analysis. The Greenhouse-Geisser correction was used when the Mauchly's sphericity test was violated. Omega squared $\left(\omega^{2}\right)$ was calculated for the ANOVA where the values of the effect sizes 0.01, 0.06 and above 0.14 were considered small, medium, and large, respectively [8]. Statistical significance was accepted at $p<0.05$ level. The JASP statistics package (version 0.11.1) was used for statistical analyses.

\section{Results}

Significant differences with a "large" effect size for the variables of $\mathrm{VM}\left(p=0.001, \omega^{2}=0.614\right), \mathrm{LC}(p=$ $\left.0.002, \omega^{2}=0.146\right)$, cadence $\left(p=0.045, \omega^{2}=0.185\right)$, support phase $\left(p=0.005, \omega^{2}=0.222\right)$, swing phase $(\%)\left(p=0.005, \omega^{2}=0.222\right)$ between the speeds. The post-hoc analysis using Bonferroni correction revealed that the VM differs in its three conditions, between $\mathrm{V} 1-\mathrm{V} 2$ (mean difference $=0.239, p=0.001$ ), $\mathrm{V} 1-\mathrm{V} 3$ (mean difference $=-0.225, p=0.004$ ) and between V2-V3 (mean difference $=-0.464, p=0.000)$. On the

Table 1. Global kinematics gait measurements obtained for the three speeds studied

\begin{tabular}{|l|c|c|c|c|}
\hline \multicolumn{1}{|c|}{ Variable } & \multicolumn{1}{c|}{ V1 } & V2 & V3 & Repeated Measures ANOVA \\
\hline $\begin{array}{c}\text { Mean (SD) } \\
(n=10)\end{array}$ & $\begin{array}{c}\text { Mean (SD) } \\
(n=10)\end{array}$ & $\begin{array}{c}\text { Mean (SD) } \\
(n=10)\end{array}$ & \\
\hline VM $[\mathrm{m} / \mathrm{s}]$ & $0.90(0.14)$ & $0.66(0.11)$ & $1.13(0.17)$ & $F(1,18)=35.3, p=0.001, \omega^{2}=0.614$ \\
\hline LC $[\mathrm{m}]$ & $0.94(0.12)$ & $0.83(0.13)$ & $1.00(0.20)$ & $F(2,18)=8.80, p=0.002, \omega^{2}=0.146$ \\
\hline Cadence [cycles/s] & $0.95(0.11)$ & $0.80(0.11)$ & $1.08(0.33)$ & $F(0.3,0.7)=4.96, p=0.045, \omega^{2}=0.185$ \\
\hline Support phase [\%] & $57.3(2.60)$ & $59.8(3.42)$ & $55.8(2.40)$ & $F(2,18)=7.16, p=0.005, \omega^{2}=0.222$ \\
\hline Swing phase [\%] & $42.6(2.60)$ & $40.1(3.42)$ & $44.1(2.40)$ & $F(2,18)=7.16, p=0.005, \omega^{2}=0.222$ \\
\hline
\end{tabular}

SD - Standard deviation, V1, V2, V3 - self-selected speed, slow and fast, respectively, VM - mean speed, LC - Stride length.

Table 2. Angular measurements included in GDI that are associated with the leveling actions of the CoM obtained for the three speeds studied

\begin{tabular}{|l|c|c|c|c|}
\hline \multicolumn{1}{|c|}{ Variable } & $\mathrm{V} 1$ & $\mathrm{~V} 2$ & $\mathrm{~V} 3$ & Repeated Measures ANOVA \\
\hline & $\begin{array}{c}\text { Mean (SD) } \\
(n=10)\end{array}$ & $\begin{array}{c}\text { Mean (SD) } \\
(n=10)\end{array}$ & $\begin{array}{c}\text { Mean (SD) } \\
(n=10)\end{array}$ & \\
\hline $\operatorname{Pr}\left[{ }^{\circ}\right]$ & $13.9(3.52)$ & $11.45(3.11)$ & $13.2(5.00)$ & $F(2,18)=1.41, p=0.268, \omega^{2}=0.001$ \\
\hline $\mathrm{Po}\left[{ }^{\circ}\right]$ & $5.08(2.72)$ & $4.01(1.74)$ & $5.4(2.75)$ & $F(2,18)=2.54, p=0.106, \omega^{2}=0.003$ \\
\hline $\mathrm{H}\left[{ }^{\circ}\right]$ & $1.94(3.45)$ & $2.87(2.10)$ & $1.74(3.07)$ & $F(2,18)=0.97, p=0.397, \omega^{2}=0.000$ \\
\hline $\mathrm{K} 1\left[^{\circ}\right]$ & $28.36(4.72)$ & $26.93(9.05)$ & $28.66(4.21)$ & $F(2,18)=0.250, p=0.780, \omega^{2}=0.000$ \\
\hline $\mathrm{K} 2\left[^{\circ}\right]$ & $64.07(5.5)$ & $60.95(7.55)$ & $62.93(7.64)$ & $F(2,18)=0.825, p=0.454, \omega^{2}=0.000$ \\
\hline Aci $\left[{ }^{\circ}\right]$ & $6.86(6.65)$ & $6.92(4.54)$ & $6.36(5.57)$ & $F(2,18)=0.051, p=0.949, \omega^{2}=0.000$ \\
\hline Am $\left[^{\circ}\right]$ & $18.4(4.6)$ & $17.7(3.7)$ & $18.3(2.5)$ & $F(2,18)=2.669, p=0.09, \omega^{2}=0.032$ \\
\hline
\end{tabular}

SD - Standard deviation, V1, V2, V3 - self-selected speed, slow and fast, respectively, Pr - Pelvic rotation range, Po - maximum pelvic obliquity, $\mathrm{H}$ - maximum adduction of hip, $\mathrm{K} 1$ - maximum knee flexion during stance, $\mathrm{K} 2$ - maximum knee flexion during swing, Aci - ankle dorsiflexion in the initial contact, Am - maximum dorsiflexion during stance. 
Table 3. Measures associated with the CoM energy transduction and $W_{\text {int }}$ obtained for the three speeds studied

\begin{tabular}{|l|c|c|c|c|}
\hline \multicolumn{1}{|c|}{ Variable } & V1 & V2 & V3 & Repeated Measures ANOVA \\
\hline & $\begin{array}{c}\text { Mean (SD) } \\
(n=10)\end{array}$ & $\begin{array}{c}\text { Mean (SD) } \\
(n=10)\end{array}$ & $\begin{array}{c}\text { Mean (SD) } \\
(n=10)\end{array}$ & \\
\hline$W_{\text {ext }}[\mathrm{J} / \mathrm{kg} \cdot \mathrm{m}]$ & $0.30(0.06)$ & $0.34(0.13)$ & $0.37(0.10)$ & $F(2,18)=1.44, p=0.261, \omega^{2}=0.021$ \\
\hline$W_{v}[\mathrm{~J} / \mathrm{kg} \cdot \mathrm{m}]$ & $0.37(0.14)$ & $0.39(0.12)$ & $0.41(0.13)$ & $F(1,10)=0.58, p=0.486, \omega^{2}=0.000$ \\
\hline$W_{h}[\mathrm{~J} / \mathrm{kg} \cdot \mathrm{m}]$ & $0.28(0.07)$ & $0.19(0.03)$ & $0.34(0.09)$ & $F(2,18)=23.9, p=0.000, \omega^{2}=0.439$ \\
\hline$W_{\text {int }}[\mathrm{J} / \mathrm{kg} \cdot \mathrm{m}]$ & $1.71(0.87)$ & $1.22(0.59)$ & $2.92(2.54)$ & $F(1,9)=4.57, p=0.057, \omega^{2}=0.135$ \\
\hline$W_{\text {tot }}[\mathrm{J} / \mathrm{kg} \cdot \mathrm{m}]$ & $2.00(0.85)$ & $1.55(0.62)$ & $3.26(2.55)$ & $F(1,9)=4.88, p=0.050, \omega^{2}=0.140$ \\
\hline$R[\%]$ & $53.02(7.50)$ & $46.04(6.03)$ & $52.14(12.6)$ & $F(2,18)=1.83, p=0.187, \omega^{2}=0.049$ \\
\hline$\%$ Cong $[\%]$ & $24.32(9.44)$ & $25.96(8.61)$ & $24.19(10.41)$ & $F(2,18)=0.10, p=0.902, \omega^{2}=0.000$ \\
\hline
\end{tabular}

SD - Standard deviation, V1, V2, V3 - self-selected speed, slow and fast, respectively, $W_{\text {ext }}$ - external mechanical work, $W_{v}$ - vertical work, $W_{h}$ - horizontal external mechanical work, $R$ - recovery, \%Cong - congruity percentage, ${ }^{*} p<0.05$, $* * p<0.01, * * * p<0.001$.

other hand, the LC is different between V1-V2 (mean difference $=0.112, p=0.001$ ) and V2-V3 (mean difference $=-0.164, p=0.019)$. The cadence only shows changes between V1-V2 (mean difference $=0.154$, $p=0.005)$, in turn, the support phase is different only between V2-V3 (mean difference $=3.99, p=0.020$ ) as well as the swing phase (mean difference $=-3.99$, $p=0.020)$ (Table 1).

No significant differences $(p>0.05)$ were found for all the angular variables associated with the vertical leveling of the CoM considered in this study between the three speeds (Table 2).

None of the pendulum-like determining variables changed except for $W_{h}$, who presented significant differences with a "large" effect size $\left(p=0.001, \omega^{2}=0.439\right)$ between the speeds. The post-hoc analysis revealed that the $W_{h}$ differs in its three speeds, V1-V2 (mean difference $0.093, p=0.005$ ), $\mathrm{V} 1-\mathrm{V} 3$ (mean difference $-0.064, p=0.015$ ), V2-V3 (mean difference -0.157 , $p=0.001)($ Table 3$)$.

\section{Discussion}

The present study was designed to analyze whether speed variations within the range that children can self-select in life diary activities, and, eventually, in an evaluation, may induce significative changes in the general kinematics of walking cycle, angular values that reducing the vertical displacement of CoM and pendulum-like determining variables.

We first verified that the speeds self-selected by the showed significant differences. Once confirmed that, the main findings found when compared the values of the variables analyzed at different speeds were: (I) the general variables used in gait evaluation (cadence, $\mathrm{LC}$, and relative duration of support and swing phases), presented differences with the speed changes. (II) No significant changes occur in angular values associated with levelling the trajectory of the CoM. (III) Regarding pendular mechanism of gait, the values usually considered ( $R$ and\% Cong) did not changed, however, significant changes with a large effect on sizes were observed between the three speeds for $W_{h}$.

In general, these results suggest that care should be taken in strict speed control when performing clinical evaluations in children aged 6-9, since variations in self-selected speed can lead to confusion in interpretation. In particular, the changes found in $W_{h}$ and the general kinematic variables, show the need for deeper approaches in order to carry out a functional evaluation of gait, considering the strategies associated with $W_{\text {ext }}$ within the cycle.

Our hypothesis was partially met. Based on what has been established in previous studies that shown that the speed at which children walk is a factor even more influential than age for gait patterns [31], we expected to observe changes in both global and angular variables. However, the changes in the former were not accompanied by significant variations in the angular values that contribute to reducing the vertical displacement CoM.

The range of self-selected speed developed by children was similar to that reported in the literature for a population with similar characteristics[2], [20]. The increase or decrease in speed can occur with changes in LC, cadence or both simultaneously. In this study, although the range of speed variation was lower than the analyzed in previous studies, children decreased and increased their speed with a change in both, particularly when a slower gait was selected.

The trend of change in the relative durations of the support and balance phases was as it happens when analyzing a greater range [33]. However, significant differences were only observed at the extremes of the 
range selected by the children (between V2 and V3). So, the possibility of changes in LC, cadence and duration of phases, should be considered in clinical evaluations or studies of gait in children when performed with self-selected speed.

Although, based on the changes observed in the global variables, we could expect alterations in angular variables, none of the angular measurements included in GDI that are associated with the leveling actions of the $\mathrm{CoM}$ that we consider presented significant changes when the children varied speed. Our results suggest that within a range of speed change that children use habitually in daily life, amplitude and shape of angular curves are not significantly altered as stated Van der Linden and colleagues [19]. Based on this result, it could be assumed that are not alterations in the vertical exursion of the CoM within the range of speeds studied.

Regarding the adjustment to the inverted pendulum model when changing the speed, all the values in table 3 were close to those reported by Schepens and colleagues [29], for the corresponding speeds and ages. It is clearly established that for all ages, the optimum speed approaches the speed at which $W_{e x t}$ is at a minimum, $R$ is at a maximum and \%Cong is at a minimum [10], [26], [33]. Although the $R$ values were a little lower than expected, this was the observed trend when comparing the values for those variables between the three speeds. However, no significant changes occurred in any case for the range of speeds developed by children. Based on this, we must strictly say that there are no changes in the work saved by mechanical energy transfer and that temporal proportion of the cycle during which the $E_{v}$ and $E_{h}$ fluctuate in the same direction it is not altered.

When comparing speeds over a wider range, at intermediate speeds, $W_{h}$ and $W_{v}$ are more or less equal. At low speeds $W_{h}$ is less than $W_{v}$, there is not enough $E_{h}$ to transform into $E_{v}$ and $R$ is low. On the contrary, at high speeds, the variations of $E_{h}$ are greater than those of $E_{v}$ and $W_{h}$ is greater than $W_{v}$. Consequently, $R$ is lower [28], [29], [33]. In this study, the range was closer to the intermediate speed, the changes in the $W_{\text {ext }}$ components were not so clear and $W_{h}$ was always lower than $W_{v}$. Of these two components, only $W_{h}$ presented significant differences when comparing the speeds, increasing together with the speed. We believe that the changes observed in $W_{h}$ indicate that at some point during the cycle the energy transfer may have been affected although $R$ and $C$ did not change. In addition, as $W_{h}$ is the work associated to the variations of Ek of the CoM in the direction of advance, we can suppose that what values associated with braking and acceleration of the body may vary. In order to discuss in more detail the relationships between angular variables and changes in the use of mechanical energy, in future work it can be interesting to use tools that allow for analyzing the actions within the cycle. This type of approach was proposed a few years ago and has been used to analyze the gait with added load by Cavagna and colleagues [7]. This approach has begun to be applied to the study of altered locomotion [1], [13].

Finally, the $W_{\text {int }}$ values in this study were always lower than $W_{\text {ext }}$. As expected, $W_{\text {int }}$ increased with the rate of progression because the faster the person walks, the more the frequency and range of movements increase [33]. This can be an important element in the evaluation of gait in children, since when increasing speed $W_{\text {int }}$ has a greater relative importance in children than in adults [15].

Some methodological limitations arose from the study and need to be further addressed. Firstly, despite sample size calculation, ten subjects may lead to type II error. On the other hand, although we consider two criteria to define the variables associated with the vertical leveling of the CoM, in the last instance, it is a subjective choice. Other angular values may be altered with changes in speed, in fact, the changes in $W_{h}$, suggest this.

\section{Conclusions}

In conclusion, when the evaluations are carried out at self-selected speed, small changes can affect the general kinematic values habitually considered in gait evaluations in children. This seems not to affect the leveling of the vertical trajectory of CoM or the pendulum mechanism, but the possible relationship between the changes observed in one of the work components and the general kinematics focus the attention and mark the need for more detailed studies in order to carry out functional evaluations that enable making more concrete interpretations of the gait alterations in children. We considered that the type of analysis performed in this work can facilitate a better understanding of how segmental impairs increase energetic demand and so these values can be useful in clinical practice.

\section{References}

[1] Balbinot G., Schuch C.P., Bianchi Oliveira H., Peyré-TARTARUGA L.A., Mechanical and energetic determinants of impaired gait following stroke: segmental work and pendular energy transduction during treadmill walking, Biol. Open., 2020, 15, 9 (7), 051581, DOI: 10.1242/bio.051581. 
[2] BennetT B.C., Russell S.D., ABel M.F., The effects of ankle foot orthoses on energy recovery and work during gait in children with cerebral palsy, Clin. Biomech., 2012, 27 (3), 287-291, DOI: 10.1016/j.clinbiomech.2011.09.005.

[3] Biewener A.A., Patterns of mechanical energy change in tetrapod gait: pendula, springs and work, J. Exp. Zool. A. Comp. Exp. Biol., 2006, 305 (11), 899-911, DOI: 10.1002/ jez.a.334.

[4] Cavagna G.A., Heglund N.C., TAylor C.R., Mechanical work in terrestrial locomotion: two basic mechanisms for minimizing energy expenditure, Am. J. Physiol., 1977, 233 (5), 243-246, DOI: 10.1152/ajpregu.1977.233.5.R243.

[5] Cavagna G.A., Kaneko M., Mechanical work and efficiency in level walking and running, J. Physiol., 1977, 268 (2), 467-481, DOI: 10.1113 jphysiol.1977.sp011866.

[6] Cavagna G.A., Saibene F.P., Margaria R., External work in walking, J. Appl. Physiol., 1963, 18, 1-9, DOI: 10.1152/ jappl.1963.18.1.1.

[7] Cavagna G.A., Willems P.A., Legramandi M.A., HEGLund N.C., Pendular energy transduction within the step in human walking, J. Exp. Biol., 2002, 205, 3413-3422.

[8] Cohen J., Statistical power analysis for the behavioral sciences, Lawrence Earlbaum Associates, 1988.

[9] Della Croce U., Riley P.O., Lelas J.L., Kerrigan D.C., $A$ refined view of the determinants of gait, Gait Posture, 2001, 14 (2), 79-84.

[10] DeJaeger D., Willems P.A., Heglund N.C., The energy cost of walking in children, Pflugers Arch., 2001, 441 (4), 538-543.

[11] Donelan J.M., Kram R., KuO A.D., Mechanical work for step-to-step transitions is a major determinant of the metabolic cost of human walking, J. Exp. Biol., 2002, 205 (23), 3717-3727.

[12] EDWARDS R.H.T., Biomechanics and energetics of muscular exercise, Muscle Nerve, 1978, 1 (2), 172, DOI: 10.1002/ mus. 880010213.

[13] FÁbrica G., Jerez-Mayorga D., Silva-Pereyra V., Pendular energy transduction in the different phases of gait cycle in post-stroke subjects, Hum. Mov. Sci., 2019, 66, DOI: 10.1016/j.humov.2019.06.006.

[14] FenN W.O., Work Against Gravity and Work Due to Velocity Changes In Running, Am. J. Physiol., 1930, 1, 93 (2), 433-462, DOI: 10.1152/ajplegacy.1930.93.2.433.

[15] Van den Hecke A., Malghem C., Renders A., Detrembleur C., Palumbo S., Lejeune T.M., Mechanical work, energetic cost, and gait efficiency in children with cerebral palsy, J. Pediatr. Orthop., 2007, 27 (6), 643-647, DOI: 10.1097/BPO.0b013e318093f4c3.

[16] JOCHYMCZYK-WOŹNIAK K., NOWAKOWSKA-LIPIEC K., ZADOŃ H., Wolny S., GziK M., GoRwa J., MichniK R., Gait Kinematics Index, Global Symmetry Index and Gait Deviations Profile: Concept of a new comprehensive methodof gait pathology evaluation, Acta Bioeng. Biomech., 2020, 22 (4).

[17] Kirmizi M., SENGul Y.S., ANGIN S., The effects of gait speed on plantar pressure variables in individuals with normal foot posture and flatfoot, Acta Bioeng. Biomech., 2020, 22, 267-282.

[18] KUO A.D., The six determinants of gait and the inverted pendulum analogy: A dynamic walking perspective, Hum. Mov. Sci., 2007, 26 (4), 617-656. DOI: 10.1016/j.humov.2007.04.003.
[19] VAN Der Linden M.L., KerR A.M., Hazlewood M.E., HiLlman S.J., RoBB J.E., Kinematic and kinetic gait characteristics of normal children walking at a range of clinically relevant speeds, J. Pediatr. Orthop., 2002, 22 (6), 800-806.

[20] Mariana HaRo D., Laboratorio de análisis de marcha y movimiento, Rev. Médica Clínica Las Condes, 2014, 25 (2), 237-247, DOI: https://doi.org/10.1016/S0716-8640(14)70034-3.

[21] Minetti A.E., Ardigò L.P., CAPOdaglio E.M., SAibene F., Energetics and Mechanics of Human Walking at Oscillating Speeds, Am. Zool., 2001, 1, 41 (2), 205-210, DOI: 10.1093/icb/ 41.2.205.

[22] Minetti A.E., Gaudino P., Seminati E., Cazzola D., The cost of transport of human running is not affected, as in walking, by wide acceleration/deceleration cycles, J. Appl. Physiol., 2013, 114 (4), 498-503, DOI: 10.1152/japplphysiol.00959.2012.

[23] Nardello F., Ardigo L.P., MinetTi A.E., Measured and predicted mechanical internal work in human locomotion, Hum. Mov. Sci., 2011, 30 (1), 90-104, DOI: 10.1016/ j.humov.2010.05.012.

[24] Perry J., AraúJo A.G.N., Schoneberger B., DE Freitas C.D., Análise de marcha, Manole, 2005.

[25] SAIBENE F., The mechanisms for minimizing energy expenditure in human locomotion, Eur. J. Clin. Nutr. England, 1990, 44, Suppl. 1, 65-71.

[26] Saibene F., MinetTi A.E., Biomechanical and physiological aspects of legged locomotion in humans, Eur. J. Appl. Physiol., 2003, 88 (4-5), 297-316, DOI: 10.1007/s00421-002-0654-9.

[27] SAunders J.B., InMAN V.T., Eberhart H.D., The major determinants in normal and pathological gait, J. Bone Jt. Surg. Am., 1953, 35 (3), 543-558.

[28] Schepens B., Bastien G.J., Heglund N.C., Willems P.A., Mechanical work and muscular efficiency in walking children, J. Exp. Biol. England, 2004 (4), 587-596, DOI: 10.1242/ jeb.00793.

[29] Schepens B., Detrembleur C., Calculation of the external work done during walking in very young children, Eur. J. Appl. Physiol., 2009, 107 (3), 367-373, DOI: 10.1007/ s00421-009-1132-4.

[30] Schwartz M.H., RozUmalski A., The Gait Deviation Index: a new comprehensive index of gait pathology, Gait and Posture, 2008, 28 (3), 351-357, DOI: 10.1016/j.gaitpost.2008.05.001.

[31] Stansfield B.W., Hillman S.J., Hazlewood M.E., Lawson A.A., Mann A.M., Loudon I.R., RobB J.E., Sagittal joint kinematics, moments, and powers are predominantly characterized by speed of progression, not age, in normal children, J. Pediatr. Orthop., 2001, 21 (3), 403-411.

[32] Willems P.A., Cavagna G.A., Heglund N.C., External, internal and total work in human locomotion, J. Exp. Biol., 1995, 198 (Pt 2), 379-393.

[33] Willems P.A., Schepens B., Detrembleur C., Marcha normal, EMC - Kinesiterapia - Med. Física, 2012, 33 (2), 1-29, DOI: https://doi.org/10.1016/S1293-2965(12)61944-6.

[34] Winter D.A., Biomechanics and Motor Control of Human Movement, Wiley, 2009.

[35] Worster K., Valvano J., Carollo J.J., Sagittal plane coordination dynamics of typically developing gait, Clin. Biomech., 2015, 1, 30 (4), 366-372, DOI: 10.1016/ j.clinbiomech.2015.02.013. 